\title{
A note on measurements
}

As historians, we use the system of currency employed by contemporaries and used until I972 in which I2 pennies (I2d) made a shilling, of which 20 made $f^{\mathrm{I}}$ (or $240 d$ ).

Land measurements are more complicated. The database is based on the measurements of land given in the survey of 1598 . These are in the form acres (a), roods (r), of which there were four in an acre, and perches (p), of which there were 40 in a rood and I6o in an acre. When we offer a figure for the acreage transferred in a copyhold transaction, we compute it by adding the acreages taken from the 1598 survey for the parcels - fields - which we believe are being conveyed. This gives us the advantage that areal measurements are in units of constant size. It brings the disadvantage that these computations are dependent on our ability to identify the land being transferred - which we believe we usually can - and that the measurements we give differ from the rounded estimates of acreage given in the court rolls themselves.

Where we have computed an acreage - say the mean acreage of land transferred by mortgage - we express it in acres and decimal parts of acres. 